\title{
Clinical Efficacy and Safety of Controlled Distraction- Compression Technique Using Expandable Titanium Cage in Correction of Posttraumatic Kyphosis
}

\author{
Dongho Kang, ${ }^{1}$ Stephen J Lewis, ${ }^{2}$ Dong-Hwan Kim ${ }^{1}$ \\ Department of Neurosurgery, School of Medicine, Gyeongsang National University, Jinju, Korea \\ Division of Orthopaedic Surgery, ${ }^{2}$ University Health Network, Toronto Western Hospital, Toronto, Canada
}

Objective : To investigate the clinical efficacy and safety of the controlled distraction-compression technique using an expandable titanium cage (ETC) in posttraumatic kyphosis (PTK).

Methods : We retrospectively studied and collected data on 20 patients with PTK. From January 2014 to December 2017, the controlled distraction-compression technique using ETC was consecutively performed in 20 patients with PTK of the thoracolumbar zone (range, 36-82 years). Among them, nine were males and 11 were females and the mean age was 61.5 years. The patients were followed regularly at 1, 3, 6, and 12 months, and the last follow-up was more than 2 years after surgery.

Results : The mean follow-up period was $27.3 \pm 7.3$ months (range, 14-48). The average operation time was $286.8 \pm 33.1$ minutes (range, 225-365). The preoperative regional kyphotic angle (RKA) ranged from $35.6^{\circ}$ to $70.6^{\circ}$ with an average of $47.5^{\circ} \pm 8.1^{\circ}$. The immediate postoperative mean RKA was $5.9^{\circ} \pm 3.8^{\circ}(86.2 \%$ correction rate, $p=0.000)$, and at the last follow-up more than 2 years later, the mean RKA was $9.2^{\circ} \pm 4.9^{\circ}(80.2 \%$ correction rate, $p=0.000)$. The preoperative mean thoracolumbar kyphosis was $49.1^{\circ} \pm 9.2^{\circ}$ and was corrected to an average of $8.8^{\circ} \pm 5.3^{\circ}$ immediately after surgery $(p=0.000)$. At the last follow-up, a correction of $11.9^{\circ} \pm 6.3^{\circ}$ was obtained $(p=0.000)$. The preoperative mean back visual analog scale (VAS) score was $7.9 \pm 0.8$ and at the last follow-up, the VAS score was improved to a mean of $2.3 \pm 1.0$ with a $70.9 \%$ correction rate $(p=0.000)$. The preoperative mean Oswestry disability index (ODI) score was $32.3 \pm 6.9(64.6 \%)$ and the last follow-up ODI score was improved to a mean of $6.85 \pm 2.9(3.7 \%)$ with a $78.8 \%$ correction rate $(p=0.000)$. The overall complication was $15 \%$, with two of distal junctional fractures and one of proximal junctional kyphosis and screw loosening. However, there were no complications directly related to the operation.

Conclusion : Posterior vertebral column resection through the controlled distraction-compression technique using ETC showed safe and good results in terms of complications, and clinical and radiologic outcomes in PTK. However, to further evaluate the efficacy of this surgical procedure, more patients need long-term follow-up and there is a need to apply it to other diseases.

Key Words : Controlled distraction · Vertebral column resection · Kyphosis · Cage, expandable.

- Received : June 14, $2021 \cdot$ Accepted : July 17, 2021

- Address for reprints : Dongho Kang

Department of Neurosurgery, School of Medicine, Gyeongsang National University, 79 Gangnam-ro, Jinju 52727, Korea

Tel : +82-55-750-8112, Fax : +82-55-759-0817, E-mail : ns4793@hanmail.net, ORCID : https://orcid.org/0000-0002-1932-5898

This is an Open Access article distributed under the terms of the Creative Commons Attribution Non-Commercial License (http://creativecommons.org/licenses/by-nc/4.0) which permits unrestricted non-commercial use, distribution, and reproduction in any medium, provided the original work is properly cited. 


\section{INTRODUCTION}

Posttraumatic kyphosis (PTK) is a complication of vertebral fracture that causes axial pain or neurological deficit due to poor reduction, unrecognized instability at the beginning of treatment, pseudarthrosis, or premature removal of the instrument $^{9,20)}$. For PTK, most of the fractures are united and stabilized, so back pain does not occur directly there. Instead, pain is often caused by compensatory lumbar hyperlordosis or early degenerative changes of the facet joints ${ }^{11)}$. In addition, pain and neurological deficits may be exacerbated due to the weakness of back muscles and an anterior shift of the line of gravity over time ${ }^{29)}$. In general, the surgical goals are to relieve pain, establish normal sagittal balance, neural decompression, and ensure a solid bony union. Among various PTK surgery, a posterior-only approach has been widely used, which is familiar to most surgeons, easily accessible, and has good recent clinical outcomes. Posterior-based osteotomy seems to be the most important technique for PTK treatment. When large correction angles of $30-40^{\circ}$ or greater are required, surgical procedures such as pedicle subtraction osteotomy (PSO), PSO variants, vertebral column resection (VCR) are used. Although these techniques can achieve a large angle of correction, but complications have been reported in $15 \%$ to $64 \%$ 1,14,18,23,27,28,33). These techniques use posterior spinal shortening as a basic mechanism for a large correction angle. Therefore, over-shorting and redundancy of spinal cord are likely to occur, resulting in neurological deficits ${ }^{3,15)}$.

When considering that one of the main goals is the correction of focal kyphosis, the surgeon must provide adequate anterior column support for the compressive forces and enough posterior instrumentation to support the correction for tensile forces and solid fusion. Without anterior support, excessive tensile forces can be placed on the posterior instrumentation, especially when the deformity consists of a focal misalignment and global sagittal balance. In such situations, the loss of fixation, implant failure, loss of correction, and pseudarthrosis often result ${ }^{4)}$.

Table 1. Patient demographics

\begin{tabular}{|c|c|c|c|c|c|c|}
\hline Case No. & Sex & Age (years) & Fx. level & Fusion level & OP time (minutes) & F/U period (months) \\
\hline 1 & $M$ & 62 & $\mathrm{~T} 12$ & T10-L2 5 & 290 & 14.0 \\
\hline 2 & M & 59 & T12, L1 & T10-L3 6 & 365 & 25.0 \\
\hline 3 & M & 55 & L1 & T11-L3 5 & 270 & 24.0 \\
\hline 4 & $\mathrm{~F}$ & 38 & L1 & T11-L3 5 & 230 & 26.5 \\
\hline 5 & M & 74 & $\mathrm{~T} 11$ & T8-L2 5 & 270 & 24.0 \\
\hline 6 & $\mathrm{~F}$ & 67 & $\mathrm{~T} 12$ & T10-L2 5 & 225 & 23.5 \\
\hline 7 & $\mathrm{~F}$ & 73 & $\mathrm{~T} 11$ & T9-L1 5 & 240 & 36.0 \\
\hline 8 & M & 73 & $\mathrm{~T} 11,12$ & T8-L2 7 & 325 & 25.0 \\
\hline 9 & M & 49 & L1 & $\mathrm{T} 11-\mathrm{L} 35$ & 310 & 48.0 \\
\hline 10 & M & 60 & $\mathrm{~T} 12$ & T9-L3 6 & 290 & 35.0 \\
\hline 11 & $\mathrm{~F}$ & 78 & L1 & T11-L3 5 & 310 & 36.0 \\
\hline 12 & $\mathrm{~F}$ & 40 & L1 & T11-L3 5 & 295 & 24.0 \\
\hline 13 & $\mathrm{~F}$ & 67 & $\mathrm{~T} 12$ & $\mathrm{~T} 10-\mathrm{L} 25$ & 265 & 24.0 \\
\hline 14 & $\mathrm{~F}$ & 38 & $\mathrm{~T} 11, \mathrm{~L} 1$ & T10-L3 6 & 315 & 24.0 \\
\hline 15 & $\mathrm{~F}$ & 36 & $\mathrm{~T} 12$ & T10-L2 5 & 285 & 24.0 \\
\hline 16 & $\mathrm{~F}$ & 61 & $\mathrm{~T} 12$ & T10-L2 5 & 290 & 24.5 \\
\hline 17 & M & 62 & $\mathrm{~T} 12$ & T10-L2 5 & 275 & 23.5 \\
\hline 18 & M & 82 & T9, 10, 11 & T8-L1 6 & 315 & 36.0 \\
\hline 19 & $\mathrm{~F}$ & 80 & $\mathrm{~T} 12$ & T10-L2 5 & 290 & 24.5 \\
\hline 20 & $\mathrm{~F}$ & 75 & $\mathrm{~T} 12$ & T10-L2 5 & 280 & 25.0 \\
\hline
\end{tabular}

Fx. : fracture, OP : operation, F/U : follow-up, M : male, F : female 
Hence, the authors designed modified technique for VCR with controlled distraction-compression using an expandable titanium cage (ETC; VLIFT ${ }^{\circledR}$ System; Stryker Spine, Kalamazoo, MI, USA) to reduce the complications of 3-column osteotomies and remove pain generator. The authors also described the clinical and radiological results of the technique in PTK.

\section{MATERIALS AND METHODS}

This study was approved by the Institutional Review Board of Gyeongsang National University (IRB No. GNUH 2020-11013-001). The study was conducted with informed consent from all the patients included in the study.

\section{Patient population}

Twenty consecutive patients diagnosed with posttraumatic thoracolumbar kyphosis in which the minimal kyphotic angle was $30^{\circ}$ were included in this study from January 2014 to December 2016 (Tables 1 and 2). There were nine males and 11 females with a mean age of 61.5 years (range, 36-82). The chief complaints of the patients were intractable pain interrupting daily life, stooping with cosmetic problems, and the inability of patients to lie down on their back due to a back prominence. Three patients showed preoperative low extremity weakness. The fracture levels were mainly in the thoracolumbar region (T9-L2). Back pain intensity was assessed by the visual analog scale (VAS). Clinical outcomes were measured by the Oswestry disability index (ODI). Neurological status was evaluated by the Frankel grade. All clinico-neurological evaluation was performed preoperatively and one year after surgery. Radiologic evaluations were performed preoperative-

Table 2. Clinical and radiological outcomes

\begin{tabular}{|c|c|c|c|c|c|c|c|c|c|c|c|c|c|c|}
\hline \multirow{2}{*}{$\begin{array}{r}\text { Case } \\
\text { No. }\end{array}$} & \multicolumn{2}{|c|}{ Preop $\left({ }^{\circ}\right)$} & \multicolumn{2}{|c|}{$\begin{array}{c}\text { Immediate } \\
\text { postop }\left({ }^{\circ}\right)\end{array}$} & \multicolumn{2}{|c|}{ Last F/U $\left(^{\circ}\right)$} & \multicolumn{2}{|c|}{ Low back ODI } & \multicolumn{2}{|c|}{ Back VAS } & \multicolumn{2}{|c|}{$\begin{array}{l}\Delta \text { (preop RKA - } \\
\left.\text { postop RKA) ( }{ }^{\circ}\right)\end{array}$} & \multicolumn{2}{|c|}{ Frankel grade } \\
\hline & RKA & TLK & RKA & TLK & RKA & TLK & Pre op & Last F/U & Pre op & Last F/U & Immediate & Last F/U & Pre op & Last F/U \\
\hline 1 & 45.0 & 46.0 & 0.0 & 14.0 & 15.0 & 16.0 & 35 & 4 & 8 & 1 & 45.0 & 30.0 & E & $E$ \\
\hline 2 & 70.6 & 75.0 & 6.0 & 6.0 & 14.6 & 14.0 & 45 & 13 & 9 & 4 & 64.6 & 56.0 & C & $E$ \\
\hline 3 & 45.0 & 51.0 & 2.0 & 10.0 & 2.4 & 15.0 & 24 & 4 & 7 & 1 & 43.0 & 42.6 & $E$ & $E$ \\
\hline 4 & 46.0 & 48.0 & -1.0 & -5.0 & 2.8 & -2.8 & 34 & 6 & 8 & 2 & 47.0 & 43.2 & $E$ & $E$ \\
\hline 5 & 35.6 & 38.4 & 11.3 & 11.7 & 13.2 & 14.5 & 33 & 5 & 8 & 2 & 24.3 & 22.4 & $E$ & E \\
\hline 6 & 43.0 & 40.0 & 5.0 & 5.5 & 8.0 & 5.5 & 27 & 5 & 7 & 2 & 38.0 & 35.0 & $E$ & $E$ \\
\hline 7 & 45.2 & 45.3 & 10.4 & 15.2 & 12.3 & 20.2 & 33 & 6 & 7 & 2 & 34.8 & 32.9 & $E$ & $E$ \\
\hline 8 & 51.4 & 56.0 & 10.0 & 15.6 & 15.0 & 17.8 & 32 & 7 & 8 & 2 & 41.4 & 36.4 & $B$ & $D$ \\
\hline 9 & 41.5 & 47.8 & 7.7 & 8.0 & 9.2 & 10.5 & 34 & 7 & 8 & 2 & 33.8 & 32.3 & $E$ & $E$ \\
\hline 10 & 42.7 & 37.0 & 6.8 & 6.4 & 10.0 & 12.1 & 41 & 12 & 9 & 3 & 35.9 & 32.7 & $E$ & $E$ \\
\hline 11 & 47.5 & 49.0 & 0.0 & 5.3 & 2.0 & 7.3 & 30 & 8 & 8 & 2 & 47.5 & 45.5 & $E$ & $E$ \\
\hline 12 & 42.8 & 49.5 & 5.2 & 5.0 & 7.0 & 5.7 & 25 & 3 & 7 & 1 & 37.6 & 35.8 & $E$ & $E$ \\
\hline 13 & 43.0 & 40.0 & 6.3 & 5.5 & 8.0 & 5.5 & 23 & 4 & 7 & 1 & 36.7 & 35.0 & $E$ & $E$ \\
\hline 14 & 54.1 & 54.1 & 0.0 & 5.0 & 0.5 & 7.0 & 32 & 6 & 8 & 2 & 54.1 & 53.6 & $E$ & $E$ \\
\hline 15 & 45.0 & 46.0 & 11.0 & 14.0 & 15.0 & 16.0 & 34 & 5 & 8 & 4 & 34.0 & 30.0 & $E$ & $E$ \\
\hline 16 & 38.8 & 41.2 & 5.0 & 11.6 & 7.0 & 13.4 & 26 & 5 & 7 & 2 & 33.8 & 31.8 & $E$ & $E$ \\
\hline 17 & 45.8 & 47.0 & 8.3 & 8.4 & 10.4 & 15.3 & 28 & 6 & 8 & 2 & 37.5 & 35.4 & $E$ & $E$ \\
\hline 18 & 50.0 & 48.0 & 13.6 & 17.4 & 17.0 & 25.4 & 24 & 10 & 7 & 4 & 36.4 & 33.0 & $E$ & $E$ \\
\hline 19 & 62.0 & 65.0 & 4.5 & 11.2 & 10.0 & 12.0 & 46 & 10 & 9 & 3 & 57.7 & 52.0 & $C$ & $D$ \\
\hline 20 & 55.0 & 58.6 & 3.0 & 4.6 & 5.2 & 6.8 & 42 & 11 & 9 & 3 & 52.0 & 49.8 & $E$ & $E$ \\
\hline
\end{tabular}

RKA : regional kyphotic angle, TLK : thoracolumbar kyphosis, F/U : follow-up, ODI : Oswestry disability index, VAS : visual analog scale 
ly, immediately after surgery, 3 months, 6 months, 1 year, and more than 2 years postoperatively.

\section{Radiographic measurements}

The whole spine of the patient was evaluated by a standing $\mathrm{X}$-ray that included anteroposterior and lateral views with visualization from the $\mathrm{C} 7$ vertebral body (VB) to the pelvis to evaluate global sagittal balance and compensatory curve. We also checked the dynamic view, and supine lateral view to evaluate the flexibility of the deformed spine for preoperative planning.

We measured the regional kyphotic angle (RKA) and thoracolumbar kyphotic angle (TLK) using Cobb's method. The C7 sagittal vertical axis (SVA) and lumbar lordosis (LL) are also measured by the standard measurement technique. RKA was checked by Cobb's angle between the upper endplate of one vertebra above the fractured vertebra and the lower endplate of one vertebra below the fractured vertebra. The deformity correction rate was calculated as [(preoperative RKA postoperative RKA) / preoperative RKA] × 100\%.

Preoperative magnetic resonance imaging was routinely performed to exclude cord compression and intraspinal pathologies. We also performed 3-dimensional reconstruction computed tomography (CT) scanning not only for deformity assessment including the bony fusion state at the index level and around the bone preoperatively but also for the assessment of bone fusion during follow-up.

\section{Surgical technique}

All surgeries were performed by a single surgeon. Under general anesthesia, each patient was placed in the prone position with the extension of the hip and knee. All patients underwent intraoperative neuromonitoring (IONM) including somatosensory-evoked potential, motor-evoked potential, and free-running electromyography.

The overall surgical procedures were performed sequentially as follows : a standard midline incision was made with subperiosteal dissection extending to the transverse process (TP) and the medial rib portion, which was planned as a costotransversectomy. Pedicle screw fixation was performed from two or three levels above and below the level of the corpectomy according to bone quality (Fig. 1A). Additionally, we performed cement-augmented pedicle screw fixation in cases of poor bone quality and osteoporosis. Wide laminectomy, face- tectomy, transversectomy with bilateral foraminotomy checking nerve root were done. Laminectomy was performed from the pedicle one level above to the disc space one level below at the site where the corpectomy was to be performed (Fig. 1BD). The superior articular process one level below the corpectomy site was removed to secure for discectomy of the level below. For thoracic lesions, the degree of resected ribs depended upon whether an ETC could be placed into the corpectomy site. Mostly, the affected rib was transected about $1-1.5 \mathrm{~cm}$ from the lateral border of the TP, unlike conventional surgery which removes the ribs $5-6 \mathrm{~cm}$ (Fig. 2). After the ipsilateral pediculectomy, the unilateral dissection of only one-third to one-half of the lateral aspect of $\mathrm{VB}$ was done unlike conventional surgery to dissect the entire lateral vertebral wall (Fig. 2). Partial discectomies above and below the corpectomy site were done with pituitary forceps to mark the corpectomy limits. We started the corpectomy cross midline while dissecting the ventral portion of the dura and VB in piecemeal fashion using an osteotome, rongeur, and pituitary forceps instead of a high-speed drill and curette as in other procedures (e.g., eggshell osteotomy). Only one-third to one-half of the lateral wall of the VB was removed to secure enough space for the ETC (Fig. 2). After control of bone bleeding with hemostatic materials, contralateral transversectomy, partial pediculectomy, and corpectomy leaving the $\mathrm{VB}$ wall intact were performed with applying a temporary rod to the contralateral side. In general, fibrous tissue is formed in the front of the fractured vertebrae, so removing this fibrous tissue helps to correct kyphosis. However, we should be careful because removing too much can damage blood vessels. And then, removal of the remnant posterior $\mathrm{VB}$ wall under the dura was performed with a reverse-angled curette (Fig. 1E). Since there are often a high degree of adhesion between the posterior VB wall and posterior longitudinal ligament, it must be carefully peeled off not to tear dura. Then, the remaining discs above and below the corpectomy site should be carefully removed without damage to the bony endplate. After completion of the vertebral resection, we exchanged the short temporary rod for a pre-bent and suitably sized permanent rod (Fig. 1I). ETC filled with autologous and allogenic bone was inserted into the empty space in the collapsed state (Fig. 1G). After placement, we expanded the ETC along with sagittal alignment until the anterior vertebral height was nearly similar to the posterior vertebral height with the temporary rod fixed (Fig. 1H). Once 
the cage was expanded, a large amount of additional bone chips were packed into the expanded cage and between the ETC and residual cortical shell (Fig. 1J and K). We did not routinely sacrifice the thoracic nerve roots if they were not severely disturbed when performing corpectomy or ETC insertion. For lumbar lesions, the ETC can be inserted in a direc- tion perpendicular to the dura and rotated to expand (Fig. 3) because nerve roots often interfere with ETC insertion. Finally, the correction is completed by posterior compression (Fig. 1I).
(A)

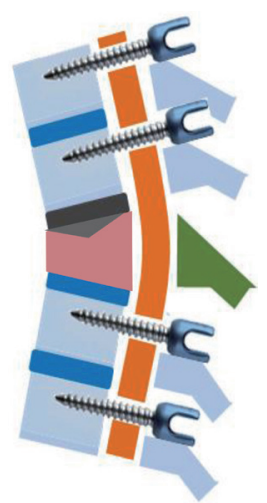

(E)

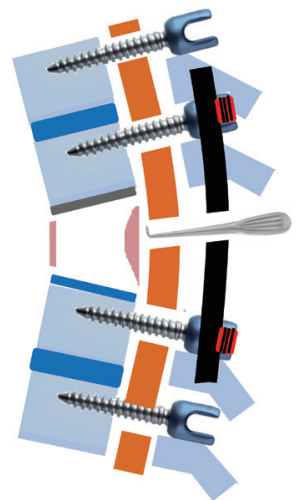

(1)

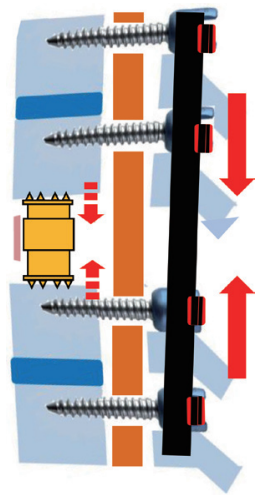

(B)

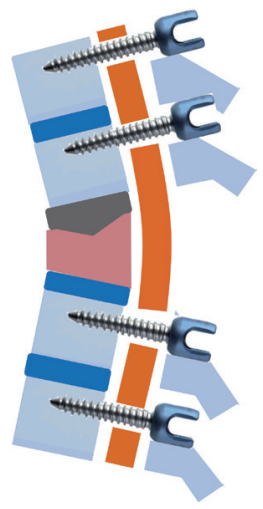

(F)
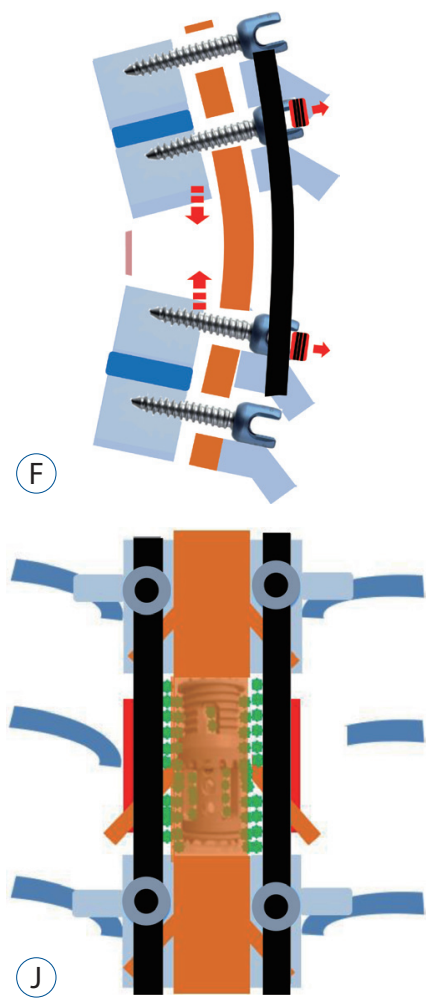

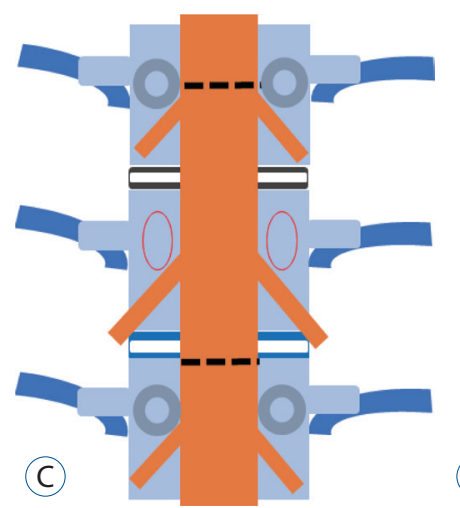

(D)

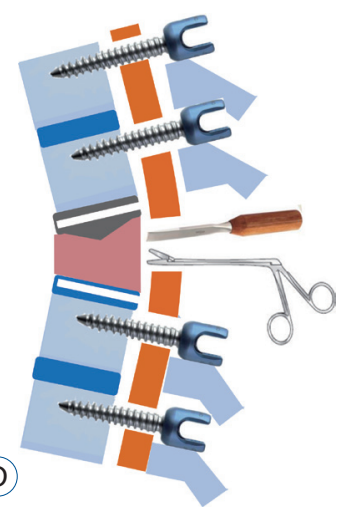

(G)

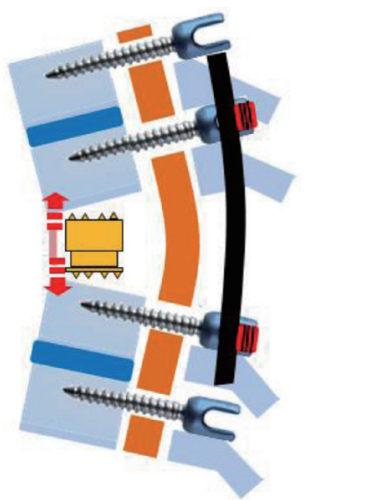

(H)

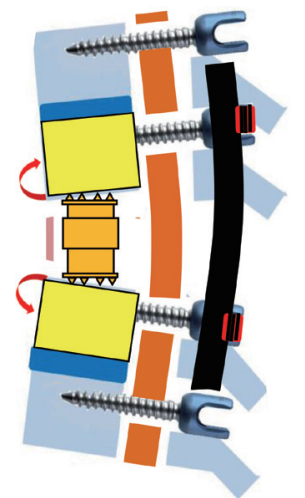

(K)

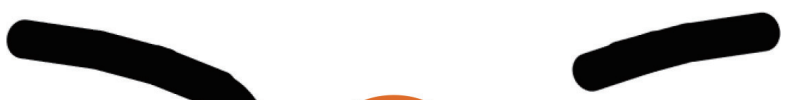

Fig. 1. Pedicle screw fixation (A), and laminectomy and its limits (B and C). Laminectomy extent is between dashed lines (C). Partial discectomy and corpectomy with an osteotome and pituitary forceps (D), and removal of the remnant posterior vertebral body wall under the dura done with a reverseangled curette (E). If the cap of the temporary rod was loosened temporarily before inserting the expandable titanium cage, the partial reduction of kyphosis by gravity could be confirmed (F). An expandable titanium cage (ETC) was inserted into the empty space in a collapsed state (G). After placement, we expanded the ETC along with sagittal alignment until the anterior vertebral height was nearly similar to the posterior vertebral height with a temporary rod fixed $(\mathrm{H})$. The short temporary rod was exchanged for a pre-bent and suitably sized permanent rod (I). Once the cage was expanded, a large amount of additional allogenous and autogenous bone chips were packed into the expanded cage and between the ETC and residual cortical shell ( $\mathrm{J}$ and $\mathrm{K}$ ). 


\section{Statistical analysis}

We used analysis of variance to compare the preoperative and postoperative measurements in each group after consulting with a statistician. For the continuous variables, ANOVA
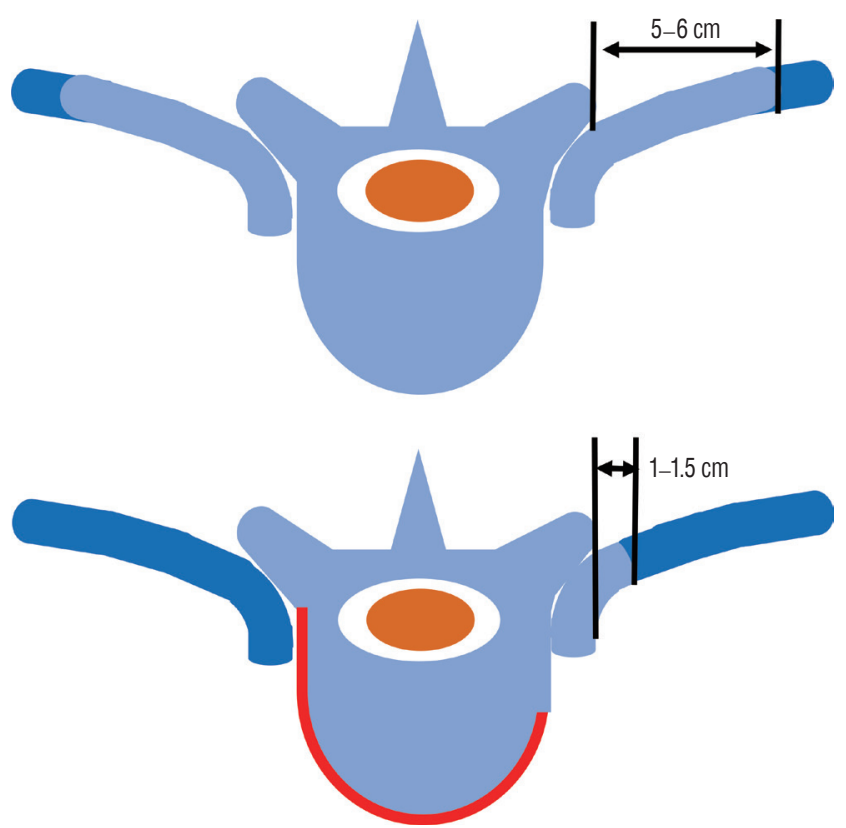

Fig. 2. Comparison of the extent of bone removal. The affected rib was transected about $1-1.5 \mathrm{~cm}$ from the lateral border of the transverse process (TP) (lower), unlike conventional surgery which removes ribs 5-6 $\mathrm{cm}$ from the lateral border of the TP (upper). Only one-third to one-half of the lateral wall of the vertebral body was removed to secure enough space for the expandable titanium cage. Blue color : removed bone, dark blue : rib, red : remnant cortical shell. with an unpaired t-test was used for the variables with normality, and Wilcoxon's rank-sum test was used for the variables without normality. The categorical variables were assessed using the chi-squared test, compensated using Fisher's exact test, and the linear mixed model was performed to evaluate every considerable factor. All statistical analyses were performed using R version 4.0.3 (R Core Team. R Foundation for Statistical Computing, Vienna, Austria) and the significance level was set to 0.05 .

\section{RESULTS}

One-level VCR was performed in 19 patients and 1.5-level VCR in one patient. The mean instrumented fusion levels were 5.3 levels (range, 5-7). In the case of poor bone quality, we used alternative techniques including hook, instrumentation extension, or cement-augmented screw fixation to enhance the pull-out strength. The mean follow-up period was $27.3 \pm 7.3$ months (range, 14-48). The mean operation time was 286.8 \pm 33.1 minutes (range, 225-365). The pain intensity, clinical outcome, and neurological status significantly improved compared to the preoperative status. The preoperative mean back VAS score was $7.9 \pm 0.8$ and the last follow-up VAS score was improved to a mean of $2.3 \pm 1.0$ with a $70.9 \%$ improvement $(p=0.000)$.

The preoperative mean ODI score was $32.3 \pm 6.9$ (64.6\%) and the last follow-up ODI score was improved to a mean of $6.85 \pm$
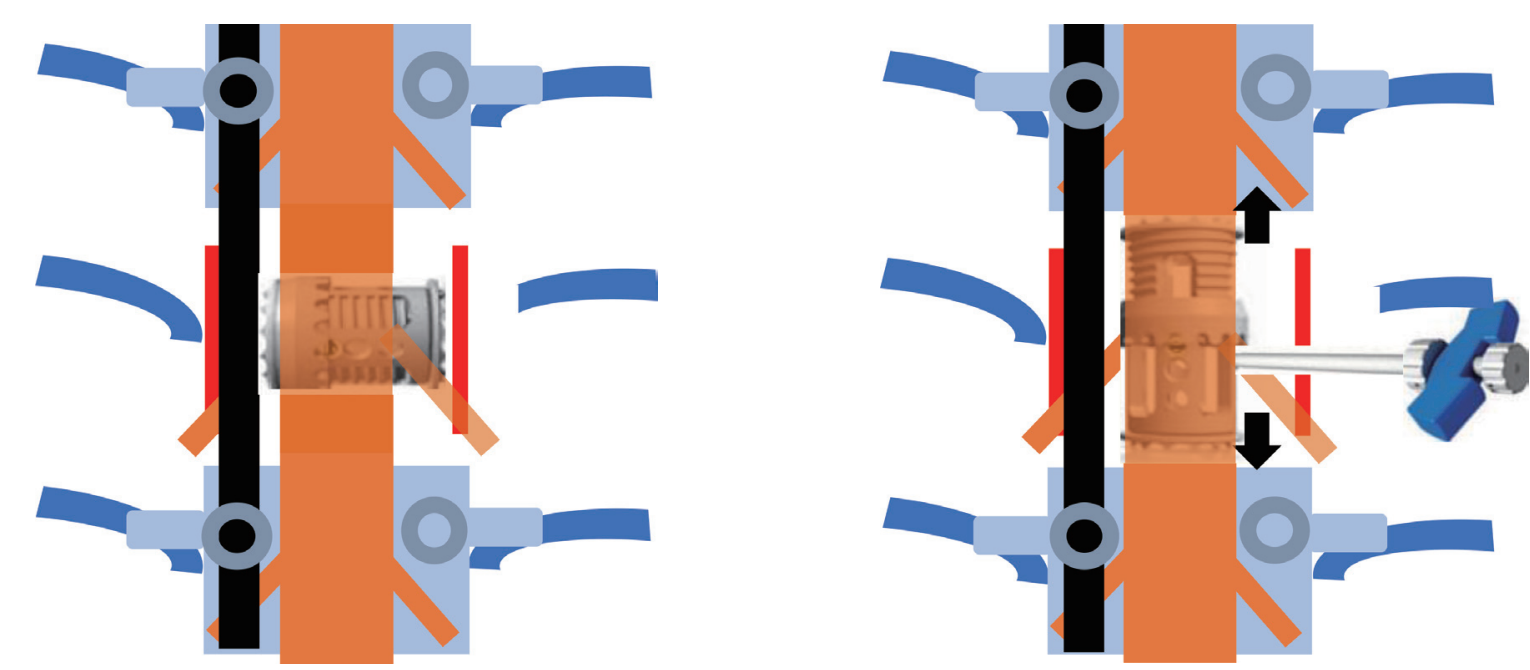

Fig. 3. The expandable titanium cage can be inserted into the corpectomy site in a direction perpendicular to the dura and rotated to expand for lumbar lesions. 
$2.9(13.7 \%)$ with a $78.8 \%$ improvement $(p=0.000)$. Three patients had weakness in both lower extremities and one of them had bladder symptoms. One patient recovered normal motor power, but two patients recovered to a Frankel grade of D at the last follow-up. The patient with bladder symptoms was able to self-void with less than $100 \mathrm{~mL}$ of residual urine.

The preoperative RKA ranged from $35.6^{\circ}$ to $70.6^{\circ}$ with an average of $47.5^{\circ} \pm 8.1^{\circ}$. The immediate postoperative mean RKA was $5.9^{\circ} \pm 3.8^{\circ}(86.2 \%$ correction rate, $p=0.000)$, and at last follow-up more than 2 years later, it was $9.2^{\circ} \pm 4.9^{\circ}(80.2 \%$ correction rate, $p=0.000$ ) with a mean loss of correction of $3.3^{\circ}$. The preoperative mean TLK was $49.1^{\circ} \pm 9.2^{\circ}$ and was corrected to an average of $8.8^{\circ} \pm 5.3^{\circ}$ immediately after surgery $(p=0.000)$. At the last follow-up, a correction of $11.9^{\circ} \pm 6.3^{\circ}$ was obtained ( $p=0.000)$, but a mean loss of correction of $3.1^{\circ}$ was seen.
Although whole spine X-rays could not be performed in all patients due to weakness and severe pain, anecdotally, there was no significant change in the C7 SVA before and after surgery, but LL was decreased after surgery. Most patients seemed to have focal thoracolumbar deformities with compensatory mechanisms rather than global sagittal imbalance. Solid bone fusion was confirmed on the dynamic view and CT the second year after surgery in all patients except one (case 2). The overall fusion rate was 95\%. The patient in case 2 was a cancer patient who died of the progression of non-small cell lung cancer 14 months after surgery.

There were three complications, for a 15\% overall complication rate. Two patients (cases 2 and 10) had distal junctional fractures (DJFs) at 3 and 5 months after surgery, respectively. One patient (case 2, Fig. 4) had severe osteoporosis (bone min-
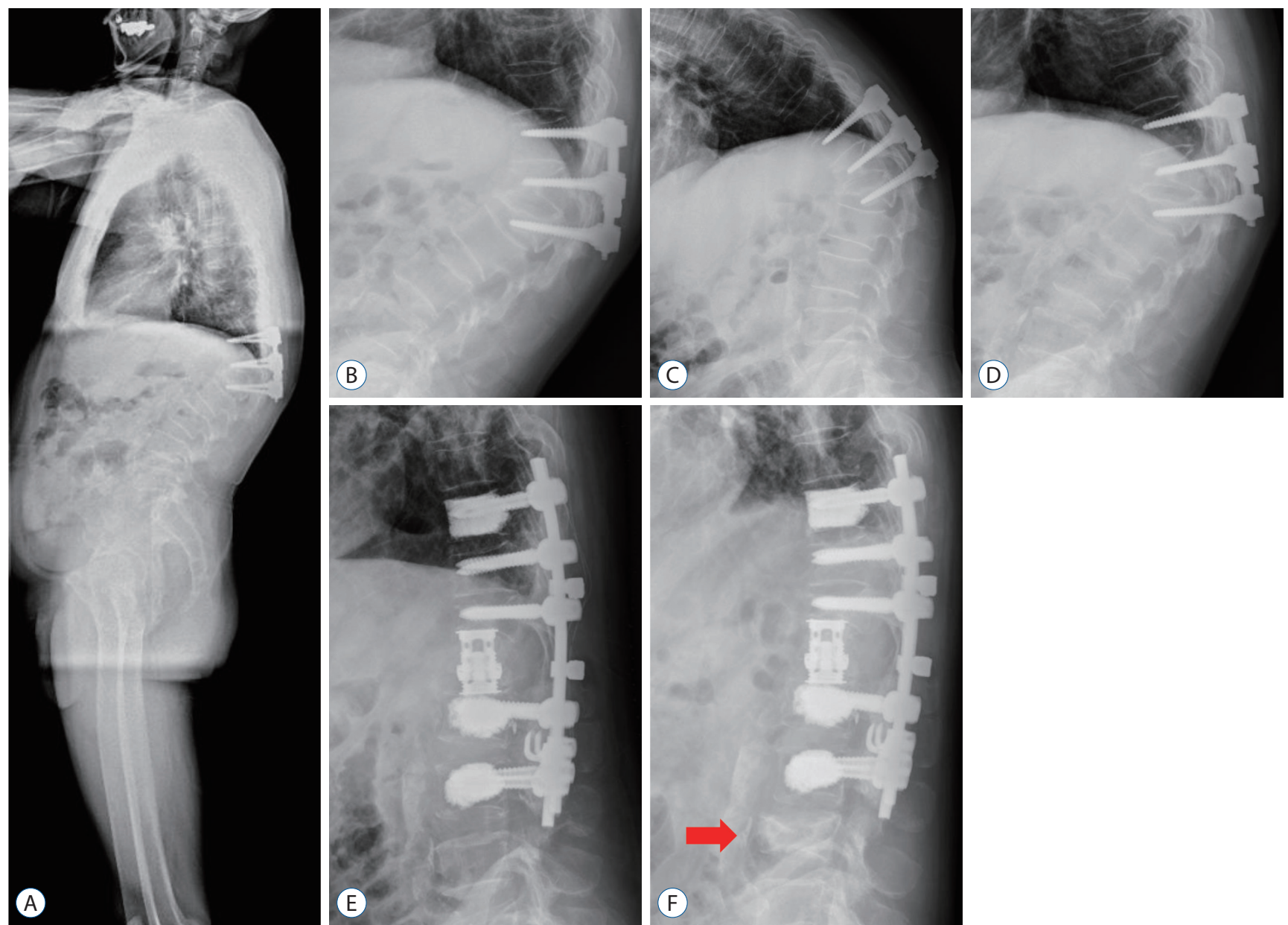

Fig. 4. Case 2. The whole spine X-ray (A) shows focal kyphosis in the thoracolumbar junction without global sagittal imbalance. The dynamic views (BD) show rigid kyphosis with a $70^{\circ}$ regional kyphosis angle (RKA) and $67^{\circ}$ of thoracolumbar kyphosis (TLK). All RKA and TLK were improved to $6^{\circ}$ immediate postoperatively (E). Although one patient developed a distal junctional fracture 3 months postoperatively ( $F$, red arrow), the patient recovered well with conservative treatment. 
eral density [BMD], -3.8) and was a cancer patient. We treated his new fracture with an additional Thoraco Lumbo Sacral Orthosis brace and pain control. The other patient (case 10) incurred a fracture after a fall and was also treated conservatively. One patient (case 18) had proximal junctional kyphosis (PJK) 6 months postoperatively and showed a halo sign of the T8 screw and T7-8 subluxation. He was older and had osteoporosis (BMD, -3.0) and weak back muscles. We revised this patient with a proximal fusion extension to T5. Apart from these, there were no direct surgery-related complications including spinal cord injury (SCI), deep or superficial wound infections, hemopneumothorax, dural tears, or cerebrospinal fluid leakage.

\section{DISCUSSION}

In PTK, abnormal vertebral alignment due to posterior deformation also changes the length and tension of the paraspinous muscle, leading to fatigue and inflammation, resulting in intractable back pain ${ }^{26,35)}$. Several authors reported that a deformity of $30^{\circ}$ or more had an increased risk of chronic pain or progression ${ }^{6}$. Considering the pathophysiology of PTK, we performed surgical treatment for severe intractable pain despite conservative treatment, neurological deficit, and poor functional intolerance in PTK patients with a thoracolumbar junction of at least $30^{\circ}$ to correct kyphosis and alter the biomechanics of the spine around the deformation.

Kyphosis correction through the anterior approach is often difficult and disturbed by posterior structures ${ }^{16)}$ and is associated with a high rate of pseudarthrosis and loss of correction $^{19)}$. However, a combined anterior and posterior approach is related to a high incidence of postoperative complications. Therefore, it would be better used in cases of revision and pseudarthroses and pathologies across the lumbosacral junction $^{2)}$. Because of these problems, surgeons usually perform posteriorly pedicle-based osteotomy in PTK surgery with decompression, kyphosis correction, and realignment. Osteotomies, particularly posteriorly based pedicle subtraction techniques, seem to be the most important modalities in the treatment of $\mathrm{PTK}^{16,21)}$. Posterior pedicle-based osteotomies including PSO, VCR, and their modifications enabled simultaneous bi-directional access to the pathological location by only a posterior approach. And, it is safe because the surgeon can operate by directly seeing and confirming the healed bone fragments compressing the nerve and spinal cord. It is more advantageous for kyphosis correction by removing posterior structures such as a fused facet joint or fusion mass and accomplishing multiple posterior stabilizations ${ }^{10,16)}$. Especially, in the case of VCR, correction rates of about $50-70 \%$ can be obtained even in patients with severe kyphoscoliosis with both coronal and sagittal deformities ${ }^{1,8,14,18,23,24,27,28,30,31,33)}$.

However, some authors have reported complication rates of 15-64\% in 3-column osteotomies ${ }^{1,14,18,23,27,28,33)}$. They have also reported that the major complication rates of nerve injuries, paraparesis and paraplegia (permanent or transient), deep infection, fatal cardiopulmonary problems, and instrumentation failure are $20-35 \%$ higher in patients who underwent 3-column osteotomies ${ }^{1,14,18,23,27,28,33)}$. Auerbach et al. ${ }^{1)}$ reported that, of the $35 \%$ of patients who had major complications, $38 \%$ had undergone PSO and 22\% had VCR. Kim et al. ${ }^{18)}$ reported that the major complication rates of posterior vertebral column resection (PVCR) and PSO were 32\% and 30\%, respectively, the minor complication rates of PVCR and PSO were $14 \%$ and $15 \%$, respectively.

Of these reported complications, neurological complications due to temporary or permanent SCI are the more important because they directly affect the prognosis of the patient. The mechanism for kyphosis correction in posterior pedicle-based osteotomies is based on posterior shortening after osteotomy or vertebrectomy. Therefore, there is always a risk of SCI due to over-shortening and redundancy of the spinal cord.

Some animal studies reported that SCI occurred from a spinal shortening of more than one-half to two-thirds ${ }^{10,15,17)}$ or $73.8 \%$ of the vertebral body height $(\mathrm{VBH})^{25)}$. However, a clinical study by Bourghli et al. ${ }^{3)}$ reported that 10 patients treated with modified closing-opening wedge osteotomy at the TL junction and underwent shortening of the $\mathrm{VBH}$ by $35 \%$ and $38 \%$ showed transient neurological deficits postoperatively. In addition, they recommended that spinal shortening of onethird or more of the VBH at the TL junction should be avoided because of the risk of SCI. And they emphasized that the human spinal cord was less tolerable of TL junction shortening than that of animals. The risk of SCI due to 3-column osteotomies based on posterior shortening has been reported by several authors and is generally recommended to be corrected to $30-45^{\circ 7,12)}$. 
In PTK, upper endplate fractures are more common, disrupted discs penetrate the fractured gaps, and degenerative changes occur, causing chronic low back pain, instability, nonunion, and progression of the deformity. Therefore, the authors devised a surgical technique that could remove the disc causing back pain and reduce complications and prospectively applied this technique to the treatment of PTK with more than $30^{\circ}$ of kyphosis. Our technique can remove the degenerated disc causing chronic back pain and is based on an anterior controlled distraction-compression technique using ETC without shortening of the cord, such as in other 3-column osteotomies, which has a relatively high risk of SCI.

The author's surgical technique has several notable features compared to conventional PVCRs. First, our technique does not remove all the anterior and lateral walls of the $\mathrm{VB}$ like conventional PVCRs, instead, only one-third to one-half of the lateral walls of one VB was dissected and removed, enough to insert the ETC. We performed a minimal costotransversectomy that removes the rib head and about $1-1.5 \mathrm{~cm}$ of the rib from the lateral border of the TP. And, since the remaining thin rim of the cortical shell acts as a surrounding mechanical barrier for fitting and impaction of the bone graft, it is advantageous for bone union (Fig. 5). Second, there is a fundamental difference in that our surgical technique is a kyphosis correction technique accomplished mainly by anterior controlled distraction instead of correction by posterior spinal shortening. Since Wang et al. ${ }^{31)}$ published a modified PVCR technique, several authors have published additional modified PVCRs ${ }^{8,24,30)}$, but the fundamental technique of surgery is not significantly different from the posterior shortening correction method using Wang's transpedicular eggshell technique.

SCI is known to occur not only by cord shortening but also by distraction and has been reported in adolescent idiopathic scoliosis surgery and distraction SCI animal models ${ }^{5,13,32,34)}$.

Wu et al. ${ }^{32)}$ reported that no SCI occurred when distracted between L1 and L3 segments by 20\% in experiments including 32 rabbits. Considering that the length of the same segment of a human is $90-100 \mathrm{~mm}^{5}$, this is equivalent to $18-20$ $\mathrm{mm}$ in humans and is reported to be a safe zone for spinal surgery. In another group, SCI was reported in distractions of $20.2 \pm 4.7 \mathrm{~mm}$, which corresponds to $3.6 \%$ of the TL junction length and $74.3 \%$ of the segmental vertebral height, in the global osteotomy of 3 -column pig models ${ }^{13,34)}$. Thus, in some animal experiments, the cutoff value for distraction SCI in humans can be approximated to about $20 \mathrm{~mm}$, but it is not possible to directly compare animal cords to those of humans. Furthermore, no paper has suggested a definite extents of distraction that is safe in clinical practice, and further research on this is needed in the future.

As seen in animal studies, cords are very vulnerable to distraction injury. Therefore, the authors used a controlled distraction technique using an ETC as a defense mechanism to SCI resulting from excessive distraction. In most cases, the ETC is made to the minimum size to insert into the vertebrectomy site and distracted under fluoroscopic guidance. If the cap of the temporary rod is loosened temporarily before inserting the ETC, partial reduction of the kyphosis by gravity can be confirmed (Fig. 1F). To prevent over-distraction and translation of the cord, the temporary rod on the opposite side of the ETC insertion was fixed and distraction was performed until the modular end caps on both sides were touched on the bony endplate. Afterward, further distraction was done until firm resistance is felt at both bony endplates. The authors set the distraction level of the anterior vertebral height within a range not exceeding the posterior vertebral height to minimize cord distraction because the anterior column is in a
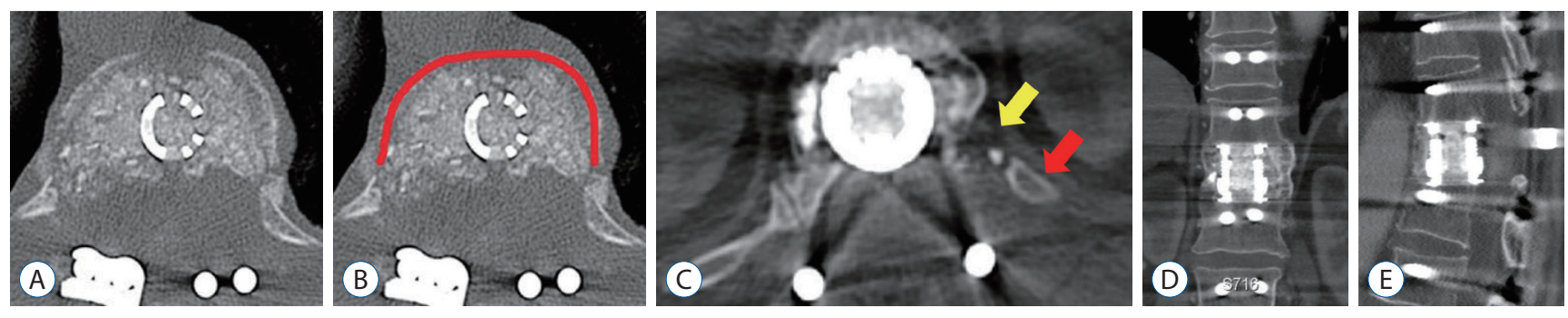

Fig. 5. Immediate postoperative computed tomography ( $C T$; $A$ and $B)$. The remaining thin rim of the cortical shell acts as a surrounding mechanical barrier for fitting and impaction of the bone graft and is advantageous for bone union (A and B). Last follow-up CT shows fused state of corpectomy site $(C-E)$. The red line $(B)$ indicate the remaining cortical rim and the yellow arrow indicates the resected rib and the red one is the remnant rib. 
shortened state and the posterior column is in a relatively normal state in PTK. After that, a permanent rod was applied to the ipsilateral side and the screw head was compressed for further kyphosis correction. Finally, the opposite temporary short rod was replaced with a permanent rod and then compressed so that the upper and lower end plates were parallel. (Fig. 1I). The entire procedure was performed with IONM. In this study, there was no change in IONM in any patients during the operation.

Although the main kyphosis correction is caused by anterior distraction, this alone in most cases does not completely correct the kyphosis. Thus, posterior compression is needed not only for this purpose but also to offset a possible distraction force to the cord to some extent. With this technique, we were able to obtain a mean kyphosis correction angle of $5.9^{\circ} \pm$ $3.8^{\circ}$ immediately after surgery and $9.2^{\circ} \pm 4.9^{\circ}$ at the last followup, which was $47.5^{\circ} \pm 8.1^{\circ}$ before surgery. The mean RKA correction rates were $86.2 \%$ and $80.2 \%$, respectively.

Some authors ${ }^{4,22)}$ reported that the prevention of kyphosis recurrence by stabilization of the vertebral column through sound bone union is more important than the amount of kyphosis correction. Generally, to achieve bone fusion with kyphosis correction, which is the main goal of deformity surgery, enough anterior column support is mandatory with compressive forces and adequate posterior instrumentation to support the correction for tensile force. In this study, the bone union rate was 95\% through ETC, but a higher bone union rate would be expected when the patient who died of cancer during follow-up was excluded from the analysis. Therefore, ETC is thought to not only be the main kyphosis correction device by regulating distraction but also an effective anterior column reconstruction device. In addition, ETC simultaneously serves to prevent excessive shortening of the spinal cord.

The complication rate in this study was 15\% (two DJF, one $\mathrm{PJK})$. There were no symptoms due to SCI or nerve injury after surgery. In addition, there was no vessel injury, pleural injury with hemopneumothorax, or other complications such as deep or superficial surgical site infections. In conclusion, we could safely and effectively operate without any direct complications related to surgery.

The authors have used this surgical technique in the operation of other diseases like spinal metastasis, acute spinal trauma, and infectious spondylitis and have been able to safely accomplish decompression including the removal of pathology and anterior column reconstruction without using the anterior approach.

There were some limitations to this study including retrospective study and relative small sample size. This study was limited to PTK, most of which were one-level vertebrectomy (excluding one case) and the patients with preoperative RKA usually were not severe. Therefore, to evaluate the clinical efficacy and safety of this surgical technique more accurately, it is necessary to apply the technique to other disease groups (e.g., Pott's kyphosis and congenital kyphoscoliosis) with severe kyphosis and fusion of several segments. It is also necessary to conduct a comparative study with conventional VCR and further research in this area is planned.

\section{CONCLUSION}

PVCR through the controlled distraction-compression technique using an ETC showed safe and good results in terms of complications, clinical, and radiological outcomes in PTK. However, to further evaluate the efficacy of this surgical procedure, more patients with long-term follow-up and the application to other diseases are needed.

\section{CONFLICTS OF INTEREST}

No potential conflict of interest relevant to this article was reported.

\section{INFORMED CONSENT}

Informed consent was obtained from all individual participants included in this study.

\section{AUTHOR CONTRIBUTIONS}

Conceptualization : DK, SJL

Data curation : DK, DHK

Formal analysis : DK

Methodology : DK

Project administration : DK 


\author{
Visualization : DK \\ Writing - original draft : DK \\ Writing - review \& editing: DK, DHK
}

\section{ORCID}
Dongho Kang
https://orcid.org/0000-0002-1932-5898
Stephen J Lewis
https://orcid.org/0000-0002-9173-8443
Dong-Hwan Kim
https://orcid.org/0000-0002-7748-7262

\section{- Acknowledgements}

This work was supported by the Gyeongsang National University Fund for Professor on Sabbatical Leave, 2018.

\section{References}

1. Auerbach JD, Lenke LG, Bridwell KH, Sehn JK, Milby AH, Bumpass D, et al. : Major complications and comparison between 3-column osteotomy techniques in 105 consecutive spinal deformity procedures. Spine (Phila Pa 1976) 37 : 1198-1210, 2012

2. Berven SH, Deviren V, Smith JA, Hu SH, Bradford DS : Management of fixed sagittal plane deformity: outcome of combined anterior and posterior surgery. Spine (Phila Pa 1976) 28 : 1710-1715; discussion 1716, 2003

3. Bourghli A, Boissière L, Vital JM, Bourghli MA, Almusrea K, Khoury G, et al. : Modified closing-opening wedge osteotomy for the treatment of sagittal malalignment in thoracolumbar fractures malunion. Spine J 15 : 2574-2582, 2015

4. Buchowski JM, Kuhns CA, Bridwell KH, Lenke LG : Surgical management of posttraumatic thoracolumbar kyphosis. Spine J 8 : 666-677, 2008

5. Busscher I, Ploegmakers JJ, Verkerke GJ, Veldhuizen AG : Comparative anatomical dimensions of the complete human and porcine spine. Eur Spine J 19 : 1104-1114, 2010

6. Chang KW : Oligosegmental correction of post-traumatic thoracolumbar angular kyphosis. Spine (Phila Pa 1976) 18 : 1909-1915, 1993

7. Chang KW, Cheng CW, Chen HC, Chang Kl, Chen TC : Closing-opening wedge osteotomy for the treatment of sagittal imbalance. Spine (Phila Pa 1976) 33 : 1470-1477, 2008

8. Chikhale C, Swar A, Khurjekar K : Modified vertebral column resection technique for correcting congenital rigid angular kyphosis and its effect on functional outcomes. J Clin Diagn Res 10 : RC17-RC22, 2016

9. Cho KJ, Bridwell KH, Lenke LG, Berra A, Baldus C : Comparison of smithpetersen versus pedicle subtraction osteotomy for the correction of fixed sagittal imbalance. Spine (Phila Pa 1976) 30 : 2030-2037; discussion 2038, 2005
10. El-Sharkawi MM, Koptan WM, El-Miligui YH, Said GZ : Comparison between pedicle subtraction osteotomy and anterior corpectomy and plating for correcting post-traumatic kyphosis: a multicenter study. Eur Spine J 20 : 1434-1440, 2011

11. Glassman SD, Bridwell K, Dimar JR, Horton W, Berven S, Schwab F : The impact of positive sagittal balance in adult spinal deformity. Spine (Phila Pa 1976) 30 : 2024-2029, 2005

12. Hao CK, Li WS, Chen ZQ : The height of the osteotomy and the correction of the kyphotic angle in thoracolumbar kyphosis. Chin Med J (Engl) 121 : 1906-1910, 2008

13. Hong JY, Suh SW, Lee SH, Park JH, Park SY, Rhyu IJ, et al. : Continuous distraction-induced delayed spinal cord injury on motor-evoked potentials and histological changes of spinal cord in a porcine model. Spinal Cord 54 : 649-655, 2016

14. Hyun SJ, Rhim SC : Clinical outcomes and complications after pedicle subtraction osteotomy for fixed sagittal imbalance patients : a long-term follow-up data. J Korean Neurosurg Soc 47 : 95-101, 2010

15. Ji L, Dang XQ, Lan BS, Wang KZ, Huang YJ, Wen B, et al. : Study on the safe range of shortening of the spinal cord in canine models. Spinal Cord 51 : 134-138, 2013

16. Kawahara N, Tomita K, Baba H, Kobayashi T, Fujita T, Murakami H : Closing-opening wedge osteotomy to correct angular kyphotic deformity by a single posterior approach. Spine (Phila Pa 1976) 26 : 391402, 2001

17. Kawahara N, Tomita K, Kobayashi T, Abdel-Wanis ME, Murakami H, Akamaru $T$ : Influence of acute shortening on the spinal cord: an experimental study. Spine (Phila Pa 1976) 30 : 613-620, 2005

18. Kim SS, Cho BC, Kim JH, Lim DJ, Park JY, Lee BJ, et al. : Complications of posterior vertebral resection for spinal deformity. Asian Spine J 6 : 257-265, 2012

19. Kostuik JP, Matsusaki $H$ : Anterior stabilization, instrumentation, and decompression for post-traumatic kyphosis. Spine (Phila Pa 1976) 14 : 379-386, 1989

20. Lafage V, Schwab F, Vira S, Patel A, Ungar B, Farcy JP : Spino-pelvic parameters after surgery can be predicted: a preliminary formula and validation of standing alignment. Spine (Phila Pa 1976) 36 : 10371045, 2011

21. Lazennec JY, Neves N, Rousseau MA, Boyer P, Pascal-Mousselard H, Saillant $G$ : Wedge osteotomy for treating post-traumatic kyphosis at thoracolumbar and lumbar levels. J Spinal Disord Tech 19 : 487-494, 2006

22. Lee JH, Oh HS, Choi JG : Comparison of the posterior vertebral column resection with the expandable cage versus the nonexpandable cage in thoracolumbar angular kyphosis. Clin Spine Surg 30 : E398-E406, 2017

23. Lenke LG, Newton PO, Sucato DJ, Shufflebarger HL, Emans JB, Sponseller PD, et al. : Complications after 147 consecutive vertebral column resections for severe pediatric spinal deformity: a multicenter analysis. Spine (Phila Pa 1976) 38 : 119-132, 2013

24. Liu X, Yuan S, Tian Y, Wang L, Zheng Y, Li J : Expanded eggshell procedure combined with closing-opening technique (a modified vertebral 
column resection) for the treatment of thoracic and thoracolumbar angular kyphosis. J Neurosurg Spine 23 : 42-48, 2015

25. Modi HN, Suh SW, Hong JY, Yang JH : The effects of spinal cord injury induced by shortening on motor evoked potentials and spinal cord blood flow: an experimental study in Swine. J Bone Joint Surg Am 93 : 1781-1789, 2011

26. Oda I, Cunningham BW, Buckley RA, Goebel MJ, Haggerty CJ, Orbegoso $\mathrm{CM}$, et al. : Does spinal kyphotic deformity influence the biomechanical characteristics of the adjacent motion segments? An in vivo animal model. Spine (Phila Pa 1976) 24 : 2139-2146, 1999

27. Ozturk C, Alanay A, Ganiyusufoglu K, Karadereler S, Ulusoy L, Hamzaoglu $A$ : Short-term $X$-ray results of posterior vertebral column resection in severe congenital kyphosis, scoliosis, and kyphoscoliosis. Spine (Phila Pa 1976) 37 : 1054-1057, 2012

28. Papadopoulos EC, Boachie-Adjei O, Hess WF, Sanchez Perez-Grueso FJ, Pellisé F, Gupta M, et al. : Early outcomes and complications of posterior vertebral column resection. Spine J 15 : 983-991, 2015

29. Roberson JR, Whitesides TE Jr : Surgical reconstruction of late posttraumatic thoracolumbar kyphosis. Spine (Phila Pa 1976) 10 : 307312,1985
30. Wang H, Zhang D, Sun YP, Ma L, Ding WY, Shen Y, et al. : Unilateral posterior vertebral column resection for severe thoracolumbar kyphotic deformity caused by old compressive vertebrae fracture: a technical improvement. Int J Clin Exp Med 8 : 3579-3584, 2015

31. Wang $Y$, Zhang $Y$, Zhang $X$, Huang $P$, Xiao $S$, Wang $Z$, et al. : A single posterior approach for multilevel modified vertebral column resection in adults with severe rigid congenital kyphoscoliosis: a retrospective study of 13 cases. Eur Spine J $17:$ 361-372, 2008

32. Wu J, Xue J, Huang $R$, Zheng $C$, Cui Y, Rao $S:$ A rabbit model of lumbar distraction spinal cord injury. Spine J $16: 643-658,2016$

33. Xie J, Wang Y, Zhao Z, Zhang Y, Si Y, Li T, et al. : Posterior vertebral column resection for correction of rigid spinal deformity curves greater than $100^{\circ}$. J Neurosurg Spine $17:$ 540-551, 2012

34. Yang JH, Suh SW, Modi HN, Ramani ET, Hong JY, Hwang JH, et al. : Effects of vertebral column distraction on transcranial electrical stimulation-motor evoked potential and histology of the spinal cord in a porcine model. J Bone Joint Surg Am 95 : 835-842, S1-S2, 2013

35. Zeng Y, Chen Z, Sun C, Li W, Qi Q, Guo Z, et al. : Posterior surgical correction of posttraumatic kyphosis of the thoracolumbar segment. J Spinal Disord Tech 26 : 37-41, 2013 